\title{
Risk of Retinopathy of Prematurity in Preterm Births with Respiratory Distress Syndrome: A Population-Based Cohort Study in Taiwan
}

\author{
Ya-Wen Lin', San-Ni Chen ${ }^{2}$, Chih-Hsin Muo $\mathbb{D}^{3}$, Fung-Chang Sung ${ }^{3-5, *}$, Ming-Hung Lin $\mathbb{D}^{6,7, *}$ \\ 'School of Nursing and Graduate Institute of Nursing, China Medical University, Taichung, Taiwan; ${ }^{2}$ Department of Ophthalmology, Eye Center, China \\ Medical University Hospital, Taichung, Taiwan; ${ }^{3}$ Management Office for Health Data, China Medical University Hospital, Taichung, Taiwan; \\ ${ }^{4}$ Department of Health Services Administration, China Medical University, Taichung, Taiwan; ${ }^{5}$ Department of Food Nutrition and Health \\ Biotechnology, Asia University, Taichung, Taiwan; ${ }^{6}$ Department of Pharmacy, Chia Nan University of Pharmacy and Science, Tainan, Taiwan; \\ ${ }^{7}$ Department of Nursing, National Taichung University of Science and Technology, Taichung, Taiwan \\ *These authors contributed equally to this work
}

Correspondence: Ming-Hung Lin, Department of Pharmacy, Chia Nan University of Pharmacy and Science, No. 60, Sec. I, Erren Road, Rende District, Tainan City, 7 I7 I0, Taiwan, Email Imh.roger@msa.hinet.net; Fung-Chang Sung, Department of Public Health, China Medical University, No. I00, Sec. I, Jingmao Road, Beitun District, Taichung City, 406040, Taiwan, Email fcsung 1008@yahoo.com

Background: Respiratory distress syndrome (RDS) is a risk factor that plays an important role in retinopathy in preterm infants. We used population data of infants to investigate this relationship. This study evaluated whether respiratory distress syndrome was associated with an increased incidence of ROP in preterm infants.

Methods: From the National Health Insurance claims data of Taiwan in the 2000-2009 period, preterm infants were identified to establish a RDS cohort $(\mathrm{N}=7573)$ and a comparison cohort without RDS $(\mathrm{N}=11428)$. We followed each infant for one year to identify incident retinopathy of prematurity (ROP) for comparison between the two cohorts. The RDS cohort for comparisons of hazard ratio (HR) with $95 \%$ confidence interval (CI) were calculated.

Results: Low birth weight, an extended stay in the neonatal intensive care unit (NICU), female gender, and RDS were factors associated with an increased incidence of ROP. The ROP incidence was 2.5-fold higher in RDS children than in control (30.3 versus 11.9 per 100 person-years), with an adjusted HR (aHR) of $1.28(95 \% \mathrm{CI}=1.18-1.39)$. The incidence increased as the birth weight decreased in both cohorts, but the RDS cohort to the comparison cohort aHR decreased as the birth weight decreased, not significant for groups with birth weights less than $1500 \mathrm{~g}$. The aHR for RDS children was the highest for infants with a normal birth weight: 3.33 (95\% CI = 2.09-5.31). Among infants with RDS, the ROP incidence increased to 51.0 per 100 person-years in those who underwent invasive ventilation, or to 76.3 per 100 person-years for NICU stay for $\geq 30$ days.

Conclusion: Preterm infants with RDS are at an elevated risk of developing ROP, not limited to those with low birth weights.

Keywords: retinopathy of prematurity, low birth weight, gestational age, preterm birth, respiratory distress syndrome

\section{Introduction}

Retinopathy of prematurity (ROP) is the underdevelopment of the retina that results in disorganized growth of retinal vessels and tissues in premature infants with low birth weight associated with oxygen therapy. ${ }^{1-5}$ The underdeveloped retinas of preterm babies are predisposed to insults that interrupt neurovascular growth, resulting in ROP. The main risk factors for the development of ROP include prematurity, low birth weight (LBW), and hyperoxia. ${ }^{4-9}$ Hyperoxia is characterized by excessive oxygen supply that suppresses the growth factors once the premature infants are placed in the neonatal intensive care. Consequently, the poorly vascularized yet increasingly metabolically active retina becomes hypoxic, triggering the growth factor-induced proliferation within blood vessels, thereby increasing the risk of retinal detachment and blindness. ${ }^{9,10}$ 
Worldwide, approximately 32,000 neonates suffer from blindness or develop severe visual impairment due to ROP globally. ${ }^{10}$ The incidence of ROP continues to rise due to the increased survival rate of prematurely born infants as a result of advancement in neonatal intensive care. ${ }^{6}$ Premature infants are those born before 37 weeks of gestational age and low birth weight under 1500 grams. The incidence of ROP is also high in countries reporting high survival rates of premature infants of gestational age earlier than 30 weeks or less and extremely low birth weight of 1500 grams or less. ${ }^{6,11,12}$ For instance, in England, the annual occurrence of ROP was $1.28 \%$ in 1990 and $12.55 \%$ in 2011 in babies with a birth weight below 1500 grams. ${ }^{13}$ In the Netherlands, the occurrence of ROP in neonates with a gestational age of 32 weeks or less and/or birth weight below 1500g was $21.9 \%{ }^{14}$ (Van Sorge et al, 2014). In Iran, from 2006 to 2010, an incidence of $32 \%$ was established. ${ }^{4}$ In the United States, ${ }^{15}$ Ludwig et al noted that the occurrence of ROP increased from $14.70 \%$ in 2000 to $19.88 \%$ in 2012. Similarly, another study conducted in Turkey established an overall occurrence of type 1 ROP of $35.8 \% .{ }^{16}$ In Taiwan, ${ }^{17}$ Kang et al found the average occurrence of ROP to be $36.6 \%$. Kang et al noted that although the incidence of ROP was relatively steady from 2002 to 2011 when the study was conducted, the number of premature infants at risk of permanent visual impairment was quite high. Besides gestational age and low birth weight, other risk factors that are associated with ROP include supplemental oxygen, protracted mechanical ventilation, low Apgar score, pulmonary problems, anemia, intraventricular bleeding, necrotizing enterocolitis, and neonatal sepsis. ${ }^{17-20}$ Therefore, this study investigates the risk factors of retinopathy in preterm infants/prematurity using population data from Taiwan.

The incidence of ROP is particularly high for infants with gestation age of 30 weeks or less, or with extremely low birth weight of 1500 grams or less. ${ }^{6,11-13}$ The incidence may range from near $20 \%$ to $37 \%$ among populations of extreme low birth weights. ${ }^{14-17}$ In addition to supplemental oxygen, the development of ROP has been associated with protracted mechanical ventilation, low Apgar score, pulmonary diseases, anemia, intraventricular bleeding, necrotizing enterocolitis, and neonatal sepsis. ${ }^{17-21}$ Limited studies have reported an elevated risk of ROP for children with respiratory distress syndrome (RDS). ${ }^{6,22-24}$ The condition of RDS is a critical disorder affecting newborns, resulting from surfactant deficiency that disrupts gas exchange. ${ }^{25,26}$ RDS robs preterm infants of their first breath, and is associated with high rates of infant deaths. It is not only a threat to life, but also a risk factor for the development of complications in premature babies. Children with RDS may need extra oxygen or breathing ventilator to support their breathing. ${ }^{27,28}$ However, both RDS and protracted mechanical ventilation are risk factors for ROP. ${ }^{2,6,22-33}$ Normally, the eye may have oxygen saturation levels as high as $30-40 \mathrm{~mm} \mathrm{Hg}$ or lower, subject to the dilution factors. ${ }^{2}$ When a preterm infant with RDS is resuscitated with high oxygen concentrations, the arterial oxygen saturation is elevated, and levels of $80 \mathrm{~mm} \mathrm{Hg}$ or higher are linked to the damage of newly developed retinal capillaries. ${ }^{2}$ Moreover, once the supplemental oxygen is removed, the avascular retina of preterm infants becomes hypoxic, thereby stimulating the overexpression of angiogenic factors that are responsible for vasoproliferation observed in ROP. ${ }^{2,29}$

The objective of this study was to use population-based insurance claims data of Taiwan to compare the risk of developing ROP between preterm infants with and without RDS. This study evaluated that respiratory distress syndrome was associated with an increased incidence of ROP in preterm infants.

\section{Materials and Methods}

\section{Data Source and Study Design}

We used the longitudinal health insurance database of infants for this study, with medical claims from 1996 to 2011 for children randomly selected from all beneficiaries. The claims data included demographic status of insured individual, disease diagnosis, treatments, medications and costs of each care. Diseases were coded with the International Classification of Disease, 9th Revision, Clinical Modification (ICD-9-CM). All identification numbers were replaced with surrogate numbers for insured people to protect the privacy. This study was approved by the Research Ethics Committee of China Medical University and Hospital (CMUH104-REC2-115). The research process adhered to the principles in the Declaration of Helsinki in this study. Written informed consent from the infants was not required by the administrator of the National Health Insurance database. 


\section{Inclusion and Exclusion Criteria}

This study employed the insurance claims data consisting of medical records from 1996 to 2011 for half of all children of Taiwan. We identify preterm infants with gestational age below 37 weeks for the period from 2000 to 2009. We identified infants eligible for this study after excluding infants with missing data on sex, urbanization level of residential area, parental job, birth weight and length of stay at hospital for care (Figure 1). We stratified these infants into two cohorts based on the RDS status (ICD-9-CM 769): the RDS cohort and the non-RDS cohort (comparisons). Each infant was followed up for one year to assess the incident ROP (Figure 2).

\section{Outcome, Mechanical Ventilation Used, and RDS}

Follow-ups were conducted on all infants in both the RDS cohort and the non-RDS cohort from the date of birth until the date of ROP development, the date of withdrew from the insurance coverage, or completion of the one-year follow-up, whichever came first. The severity of ROP was classified based on no treatment (mild), laser therapy/cryotherapy (moderate), and scleral buckling/vitrectomy treatment (severe). Mechanical ventilation used and RDS were defined during the hospitalization at birth.

\section{Statistical Analysis}

Statistical analyses of the data were performed using SAS statistical package (version 9.4; SAS Institute Inc., Cary, NC, USA). We first compared the demographic status and birth weight of infants between the RDS cohort and the non-RDS

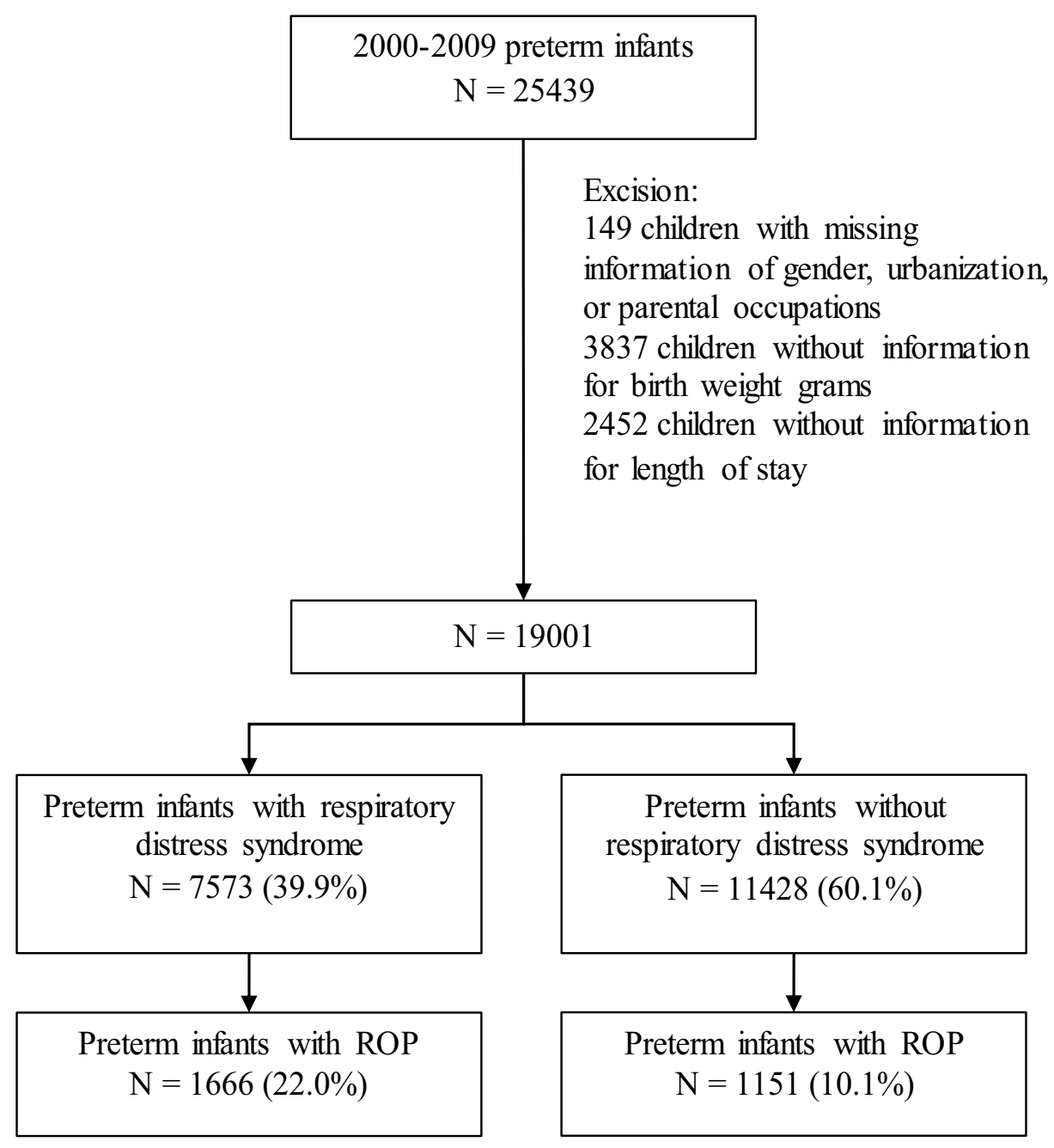

Figure I Flow chart diagram showing numbers of the enrolment. 


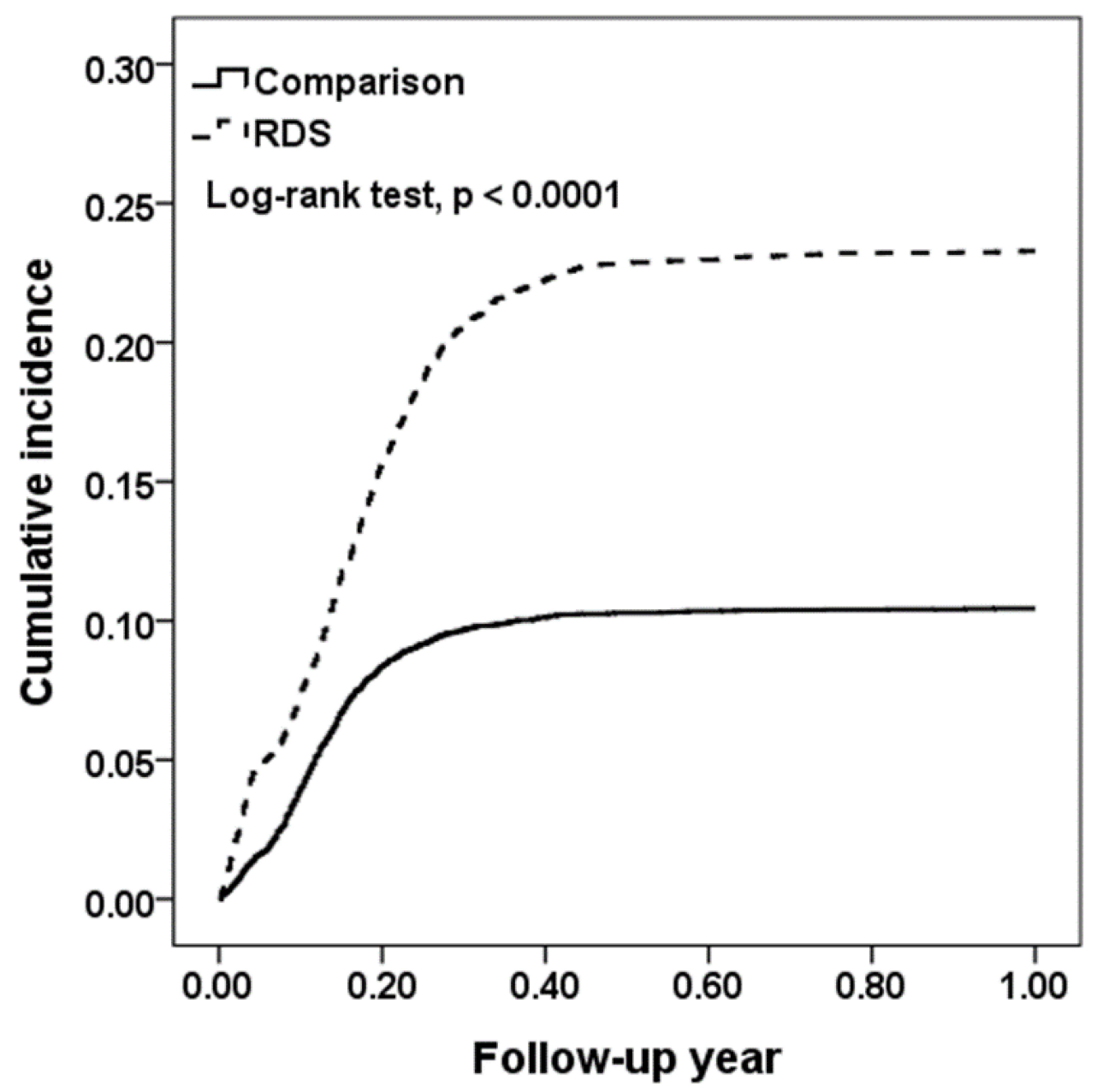

Figure 2 Kaplan-Meier cumulative incidence of ROP in preterm births compared between cohorts with and without respiratory distress syndrome.

cohort. The demographic variable included gender of babies, year of birth, urbanization level of resident and parental job type. Based on number of people living in a square kilometer, we classified urbanization level of residential areas into 4 levels; level 1 indicated the most urbanized area and level 4 the least. The urbanization level of residential area corresponded to the level of income, as parents from level 1 had the highest income. The categorical variables were presented with counts and percentages. Chi-square test was conducted to compare the difference in categorical variables between cohorts with and without RDS. We used Kaplan-Meier method to calculate and plot the cumulative incidence of ROP for both cohorts, with the difference examined by Log rank test. The incidence of ROP was calculated by dividing the sum of ROP development by the sum of follow-up person-years, presented by 100 person-years. The RDS cohort to the non-RDS cohort hazard ratio (HR) and 95\% confidence intervals (CI) were assessed using Cox proportional hazards regression analysis. The multivariable Cox model was used to estimate adjusted hazard ratio (AHR), control for sex, birth year, urbanization, parental occupations, and birth weight. To avoid the destruction of the avascular retina, infants with ROP developed may receive the treatment of peripheral retinal ablation. Data analysis also compared the ROP severity between the 2 groups based on the type of treatment received: laser therapy or cryotherapy for moderate cases and scleral buckling or vitrectomy for severe cases. A P-value of 0.05 was used as the threshold for statistical significance.

\section{Results}

In this study, 25,439 premature live births were recorded from 2000 to 2009 in the National Health Insurance claims database of children (Figure 1). After excluding 6438 preterm births with incomplete data records, 7573 infants were 
included in the RDS cohort and 11,428 in the comparison cohort, with 1666 (22.0\%) and 1151 (10.1\%) children developed ROP, respectively, in 1-year follow-up. Table 1 shows the baseline characteristics of the preterm birth cohort with and without respiratory distress syndrome. The cohort with RDS comprised 7573 infants of which 56.9 were boys. There were more infants in the cohort without RDS, 11,428, of which $54.1 \%$ were boys. Therefore, there were more boys than girls born prematurely who developed ROP. The number of premature births decreased annually from the year 2000. The births in the RDS cohort in 2000-2001 were 2515 while by the year 2008-2009 only 878 births were recorded. A similar trend is seen in the cohort without RDS where births in 2000-2001 were 4633 while by the year 2008-2009 only 745 births were recorded. The ROP incidence was higher in preterm infants born to urban or white-collar parents.

Table 2 indicates the incidence and hazard ratio of ROP in preterm births compared between cohorts with and without respiratory distress syndrome. Data from this table also indicated that boys were at a higher risk of developing ROP than girls. More male infants compared to female infants $(n=1510$ vs $n=1307)$ had ROP. This trend was consistent in both cohorts, whereby there were 908 males with ROP and 758 females with ROP in the RDS cohort, while 602 males with ROP and 549 females with ROP in the comparison cohort. The risk of ROP increased as the birth weight decreased, or as the gestational age decreased. The adjusted hazard ratio (aHR) of ROP was $3.33(95 \% \mathrm{CI}=2.09-5.31)$ for infants with

Table I Baseline Characteristics of the Preterm Birth Cohort with and without Respiratory Distress Syndrome

\begin{tabular}{|c|c|c|c|}
\hline \multirow[b]{2}{*}{ Variable } & \multirow{2}{*}{$\begin{array}{l}\text { RDS (N=7573) } \\
\text { No. (\%) }\end{array}$} & Comparison $(\mathrm{N}=|| \mathrm{428})$ & \multirow[t]{2}{*}{ P-value } \\
\hline & & No. (\%) & \\
\hline Sex & & & 0.0001 \\
\hline Girl & $3262(43.1)$ & $5249(45.9)$ & \\
\hline Boy & $4311(56.9)$ & $6179(54.1)$ & \\
\hline Birth year & & & 0.0009 \\
\hline $2000-2001$ & $2515(33.2)$ & $4633(40.5)$ & \\
\hline 2002-2003 & $2084(27.5)$ & $3930(34.4)$ & \\
\hline 2004-2005 & $1149(15.2)$ & $1266(11.1)$ & \\
\hline $2006-2007$ & $947(12.5)$ & $854(7.47)$ & \\
\hline 2008-2009 & $878(11.6)$ & $745(6.52)$ & \\
\hline Urbanization & & & $<0.0001$ \\
\hline I & $2012(26.7)$ & $3578(31.3)$ & \\
\hline 2 & $2347(31.0)$ & $3480(30.5)$ & \\
\hline 3 & $|45|(19.2)$ & $2113(18.5)$ & \\
\hline 4 & $1754(23.2)$ & $2257(19.8)$ & \\
\hline Parental occupations & & & $<0.0001$ \\
\hline White collar & $3667(48.4)$ & $6289(55.0)$ & \\
\hline Blue collar & $|36|(18.0)$ & $2045(17.9)$ & \\
\hline Others & $2545(33.6)$ & $3094(27.1)$ & \\
\hline Birth weight grams & & & $<0.0001$ \\
\hline$\geqq 2500$ & $693(9.15)$ & $2382(20.8)$ & \\
\hline $2000-2499$ & $1460(19.3)$ & $4161(36.4)$ & \\
\hline $1750-1999$ & $1152(15.2)$ & $2087(18.3)$ & \\
\hline $1500-1749$ & $1214(16.0)$ & $1312(11.5)$ & \\
\hline $1250-1499$ & $1179(15.6)$ & $826(7.23)$ & \\
\hline $1000-1249$ & $972(12.8)$ & $402(3.52)$ & \\
\hline$<1000$ & $903(11.9)$ & $258(2.26)$ & \\
\hline Comorbidity & & & \\
\hline Congenital heart disease & I357 (I7.9) & 1976 (I537) & $<0.00001$ \\
\hline Bronchopulmonary dysplasia & $531(7.01)$ & $155(1.36)$ & $<0.0000$ I \\
\hline Necrotizing enterocolitis in newborn & $282(3.72)$ & $213(1.86)$ & $<0.0001$ \\
\hline Chi-square test & & & \\
\hline
\end{tabular}


Table 2 Incidence of Retinopathy of Prematurity and Respiratory Distress Syndrome Cohort to Comparison Cohort Hazard Ratio

\begin{tabular}{|c|c|c|c|c|c|c|c|c|c|c|}
\hline \multirow[b]{2}{*}{ Demographic Factors } & \multicolumn{3}{|c|}{ Comparison Cohort } & \multicolumn{3}{|c|}{ RDS Cohort } & \multicolumn{4}{|c|}{ Hazard Ratio (95\% Confidence Interval) } \\
\hline & Event & PYs & Rate & Event & PYs & Rate & Crude & $\mathbf{p}$ & Adjusted & $\mathbf{p}$ \\
\hline Overall & $|15|$ & 9695 & 11.9 & 1666 & 5507 & 30.3 & $2.35(2.18-2.54)$ & $<0.000$ I & $1.28(1.18-1.39)$ & $<0.0001$ \\
\hline Sex & & & & & & & & & & \\
\hline Female & 549 & 4420 & 12.4 & 758 & 2328 & 32.6 & $2.39(2.15-2.67)$ & $<0.0001$ & $1.30(1.16-1.47)$ & $<0.0001$ \\
\hline Male & 602 & 5275 & 11.4 & 908 & 3179 & 28.6 & $2.33(2.10-2.58)$ & $<0.0001$ & $1.26(1.13-1.41)$ & $<0.0001$ \\
\hline \multicolumn{11}{|l|}{ Birth weight grams } \\
\hline$\geqq 2500$ & 38 & 2297 & 1.65 & 35 & 637 & 5.50 & $3.27(2.07-5.18)$ & $<0.0001$ & $3.33(2.09-5.31)$ & $<0.0001$ \\
\hline $2000-2499$ & 179 & 3831 & 4.67 & 116 & 1265 & 9.17 & I.89 (1.50-2.39) & $<0.0001$ & $1.90(1.50-2.41)$ & $<0.0001$ \\
\hline $1750-1999$ & 255 & 1695 & 15.0 & 186 & 893 & 20.8 & $1.34(\mathrm{I} . \mathrm{II}-\mathrm{I} .6 \mathrm{I})$ & 0.003 & $1.35(1.11-1.63)$ & 0.002 \\
\hline $1500-1749$ & 225 & 970 & 23.2 & 278 & 859 & 32.4 & $1.36(1.14-1.62)$ & 0.001 & $1.40(1.17-1.67)$ & 0.0002 \\
\hline $1250-1499$ & 213 & 547 & 38.9 & 329 & 796 & 41.3 & $1.04(0.87-1.23)$ & 0.672 & $1.06(0.89-1.26)$ & 0.533 \\
\hline $1000-1249$ & 125 & 241 & 51.8 & 327 & 619 & 52.9 & $1.03(0.84-1.27)$ & 0.781 & $0.99(0.80-1.22)$ & 0.905 \\
\hline$<1000$ & 116 & 114 & 101.9 & 395 & 439 & 90.0 & $0.92(0.75-1.13)$ & 0.444 & $0.91(0.74-1.12)$ & 0.380 \\
\hline
\end{tabular}

Notes: Event, number of ROP; Rate, incidence rate, per 100 person-years; Adjusted hazard ratio, adjusted for sex, birth year, urbanization, parental occupations, birth weight, and comorbidity. Abbreviations: RDS, respiratory distress syndrome; PYs, person-years. 
Table 3 Incidence and Hazard Ratio of Retinopathy of Prematurity Associated with Using Mechanical Ventilation for Infants with Respiratory Distress Syndrome

\begin{tabular}{|c|c|c|c|c|c|c|c|c|}
\hline & \multirow[t]{2}{*}{$\mathbf{N}$} & \multirow[t]{2}{*}{ Event } & \multirow[t]{2}{*}{ Person-Years } & \multirow[t]{2}{*}{ Rate } & \multicolumn{4}{|c|}{ Hazard Ratio (95\% Confidence Interval) } \\
\hline & & & & & Crude & $\mathbf{p}$ & Adjusted & $\mathbf{p}$ \\
\hline \multicolumn{9}{|l|}{ Mechanical ventilation } \\
\hline No & 1628 & 161 & 1386 & 11.62 & Reference & & Reference & \\
\hline Yes & 5945 & 1505 & 4121 & 36.5 & $2.84(2.4 \mathrm{I}-3.34)$ & $<0.0001$ & $1.82(1.53-2.15)$ & $<0.0001$ \\
\hline Noninvasive & 2402 & 381 & 1918 & 19.9 & $1.66(1.38-1.99)$ & $<0.0001$ & $1.37(1.14-1.65)$ & 0.0009 \\
\hline Invasive & 3543 & 1124 & 2201 & 51.0 & $3.75(3.18-4.22)$ & $<0.000$ I & $2.28(1.91-2.72)$ & $<0.000$ I \\
\hline \multicolumn{9}{|l|}{ Day use } \\
\hline 0 days & 1628 & 161 & 1386 & 11.6 & Reference & & Reference & \\
\hline$<14$ days & 3910 & 727 & 2916 & 24.9 & $2.05(1.73-2.43)$ & $<0.0001$ & I.75 (I.47-2.08) & $<0.0001$ \\
\hline 15-29 days & 804 & 241 & 501 & 48.1 & $3.52(2.88-4.29)$ & $<0.0001$ & $1.98(1.60-2.45)$ & $<0.0001$ \\
\hline$\geq 30$ days & $|23|$ & 537 & 704 & 76.3 & $5.04(4.23-6.01)$ & $<0.0001$ & $2.16(1.74-2.67)$ & $<0.0001$ \\
\hline
\end{tabular}

Notes: Adjusted hazard ratio, estimated controlling for sex, birth year, urbanization, parental occupations, birth weight, and comorbidity.

more than $2500 \mathrm{~g}$ birth weight. The highest incidence of ROP was recorded in infants with 1750-1999 $\mathrm{g}$ birth weight. The adjusted hazard ratio (aHR) of ROP for this group was $1.35(95 \% \mathrm{CI}=1.11-1.63)$.

Table 3 shows the incidence and hazard ratio of retinopathy of prematurity associated with the use of mechanical ventilation for infants with respiratory distress syndrome. The incidence of ROP increased with the use of mechanical ventilation. A total of 1505 ROP incidences were recorded for infants using mechanical ventilation compared to 161 ROP incidences for infants not using mechanical ventilation. There were more ROP incidences for invasive mechanical ventilation $74.7 \%$ compared to $25.3 \%$ for non-invasive mechanical ventilation. The adjusted hazard ratio (aHR) of invasive mechanical ventilation was $2.28(95 \% \mathrm{CI}=1.91-2.72)$ compared to $1.37(95 \% \mathrm{CI}=1.14-1.65)$ for non-invasive mechanical ventilation. The ROP incidence increased with longer use of mechanical ventilation. The adjusted hazard ratio (aHR) of more than 30 days use was $2.16(95 \% \mathrm{CI}=1.74-2.67)$.

Table 4 shows the severity of retinopathy of prematurity compared between study cohorts based on treatment received. Most of ROP cases in both cohorts were mild, requiring no treatment. There were more infants requiring treatment in the RDS cohort (145 moderate treatments and 18 serious treatments) than the comparison cohort (38 moderate treatments and 6 serious treatments).

\section{Discussion}

Previous study rarely investigated the ROP risk associated with new born infants with RDS. ${ }^{6,2-24}$ An earlier study in Sweden including 202 infants with birth weights of $1500 \mathrm{gm}$ and less found 81 infants developed ROP with an odds ratio of 2.0. ${ }^{22}$ Our study is the first large nation-based cohort in Taiwan population-based study that found RDS is an important factor associated with developing ROP for preterm infants. Most of ROP cases developed within 6 months after the births. However, for infants with the birth weights less than $1500 \mathrm{gm}$, the RDS cohort to the comparison cohort aHRs was not significant. The identification of 35 normal birth weight infants with ROP in the RDS cohort found that, relatively, their aHR was the highest for RDS infants, compared to non-RDS infants with normal birth weights. This finding shows that low birth weight is not the only factor leading to ROP development. Thus, screening should extend to infants with the birth weight over $1500 \mathrm{gm}$ with an unstable clinical course. The one-year follow-up revealed higher incidences of subnormal vision in preterm children. This result indicates that ROP might be associated with poorer vision later in life. Risks of developing ROP deserve attention for the normal birth weight babies with RDS.

In our study, the development of ROP was identified in the preterm births of both the RDS cohort and comparisons of non-RDS children. LBW, prolonged length of stay in the neonatal intensive care unit (NICU), and female gender were associated with increased incidences of ROP. The results obtained in this study are consistent with those reported in most other studies, ${ }^{6,12,15,17,20,31-33}$ which established that low gestational age and LBW are risk factors for the development of ROP. But, the gender differences are not the same. 
Table 4 Severity of Retinopathy of Prematurity Compared Between Study Cohorts Based on Treatment Received

\begin{tabular}{|c|c|c|c|c|c|c|c|c|c|c|}
\hline \multirow[b]{2}{*}{ Type of Treatment } & \multicolumn{3}{|c|}{ Comparison Cohort } & \multicolumn{3}{|c|}{ RDS Cohort } & \multicolumn{4}{|c|}{ Hazard Ratio (95\% Confidence Interval) } \\
\hline & Event & PYs & Rate & Event & PYs & Rate & Crude & $\mathbf{p}$ & Adjusted & $\mathbf{p}$ \\
\hline Untreated (mild) & 1107 & 9695 & 11.4 & 1503 & 5506 & 27.3 & $2.21(2.04-2.39)$ & $<0.0001$ & $1.26(1.16-1.37)$ & $<0.0001$ \\
\hline Treated with Laser therapy or cryotherapy (moderate) & 38 & 9695 & 0.39 & 145 & 5506 & 2.63 & $6.08(4.25-8.69)$ & $<0.0001$ & $1.66(1.15-2.40)$ & 0.007 \\
\hline Treated with Scleral buckling or Vitrectomy (serious) & 6 & 9695 & 0.06 & 18 & 5506 & 0.33 & $4.96(1.97-12.5)$ & 0.0007 & $1.87(0.70-4.97)$ & 0.21 \\
\hline
\end{tabular}

Notes: Event, number of retinopathy of prematurity cases; Rate, incidence rate, per 100 person-years; Adjusted hazard ratio, adjusted for sex, birth year, urbanization, parental occupations, birth weight and comorbidity. Abbreviations: RDS, respiratory distress syndrome; PYs, person-years. 
The main findings of this study were that the incidence of ROP was identified in the RDS cohort. Low gestational age, low birth weight, length of stay in the neonatal intensive care unit, male gender, and respiratory distress were associated with increased incidences of ROP. The underdeveloped retinas in preterm infants are predisposed to insults that interrupt neurovascular growth, resulting in the condition. The results obtained in this study are consistent with those reported in other studies, ${ }^{12,20,31,32}$ which established that low gestational age and low birth weight are risk factors for the development of retinopathy of prematurity.

Gestational age is the duration in weeks measured from the last day of a woman's menstruation period and can also be more accurately determined through an ultrasound. ${ }^{33}$ Preterm infants usually have a gestational age of below 37 weeks; this is a risk factor for developmental complications since the infants are born before all their tissues and organs develop fully. The gestational age at which the rate of survival is $50 \%$ is currently approximately $23-24$ weeks in developed countries. ${ }^{33}$ Low gestational age and low birth weight are directly related as infants born at low gestational age have low birth weight. From the current study, we found that the risk of ROP increased with the decrease in birth weight or the gestational age. This observation is similar to a Korean study, ${ }^{32}$ which found a high incidence of ROP in very low birth weight infants with 28 weeks and less of gestational age. ${ }^{15}$ It also found that infants with extremely low birth-weight of less than $1499 \mathrm{~g}$ were at significantly high risk of developing ROP compared to infants with normal birth weight. Lundgren et $\mathrm{al}^{31}$ found an association between low birth weight standard deviation scores and increased risk of ROP. Also, Gonçalves et $\mathrm{al}^{20}$ established in their study on the Brazilian population that birth weights of below 1000 grams and gestational age below 30 weeks were significant risk factors for the development of ROP. This study found the aHR of ROP was $1.07(95 \% \mathrm{CI}=0.90-1.27)$ for infants with birth weights of $1250 \mathrm{~g}$ to $1499 \mathrm{~g}$ while the aHR of ROP was 1.02 $(95 \% \mathrm{CI}=0.83-1.26)$ for infants with birth weights of $1000 \mathrm{~g}$ to $1249 \mathrm{~g}$. From the statistics, it is clear that the lower the birth weight or gestational age, the more is the risk of developing retinopathy of prematurity.

A longer length of stay in the neonatal intensive care unit (NICU) is significantly associated with higher odds of developing ROP. This study established more incidences of ROP $(\mathrm{n}=2192)$ for infants who had a LOS. NICU care is associated with hyperoxia, an excessive supply of oxygen that suppresses growth factors once the infants are placed there. Consequently, the poorly vascularised yet increasingly metabolically active retina becomes hypoxic, triggering the growth factor-induced proliferation within blood vessels, thereby increasing the risk of retinal detachment and blindness. ${ }^{9}$ This result is similar to that reported by Ludwig et al, ${ }^{15}$ who established that a LOS greater than 28 days had significantly increased chances of developing ROP as compared to normal birth weight. A longer length of stay indicates an unstable clinical course for premature infants indicating complications that increase the risk for ROP. ${ }^{39}$ It also found an association between longer lengths of stay in NICU and ROP, especially for preterm infants who required mechanical ventilation and oxygen therapy. Furthermore, Reyes et $\mathrm{al}^{6}$ also found that the duration of ventilation and oxygen therapy influenced the length of stay in the NICU, thereby increasing the risk of ROP.

This study revealed that boys were at a higher risk of developing ROP than girls. This finding is similar to that in the study by Lundgren et al, ${ }^{31}$ where univariate logistic regression analysis showed the male gender was at a significantly high risk of developing ROP that requires treatment. Likewise, Kang et $\mathrm{al}^{17}$ also established that one of the risk factors for ROP is the male gender. According to Lorente-Pozo et al, ${ }^{34}$ compared to their female counterparts, male infants display a lower degree of maturation that translates into a poor capacity to stabilize and, therefore, experience an increased occurrence of prematurity and prematurity-associated morbidities, and poor long-term outcomes. Male sex is an independent risk factor for major comorbidities that affect premature infants, including ROP. ${ }^{35}$

Male sex is especially linked to worse respiratory outcomes, including RDS, bronchopulmonary dysplasia (BPD), and intraventricular hemorrhage. All of these are comorbidities that increase the risk of ROP. ${ }^{36,37}$ This is because infants with respiratory distress are mechanically ventilated to receive supplemental oxygen. Normally, the eye may have oxygen saturation levels as high as $30-40 \mathrm{~mm} \mathrm{Hg}$ or lower, subject to the dilution factors. When a preterm infant with RDS is resuscitated in high oxygen concentrations, it leads to saturation in arterial oxygen. An oxygen saturation level of $80 \mathrm{~mm}$ $\mathrm{Hg}$ or more is linked to the damage of newly developed retinal capillaries. ${ }^{2}$ This study established a correlation between respiratory complications and ROP. ROP was established in preterm infants who received both invasive as well as noninvasive respiratory support. Approximately half of ROP cases occurred in preterm infants who received invasive respiratory support at birth. This finding is similar to that by Slidsborg et $\mathrm{al}^{38}$ who established mechanical ventilation 
as a significant risk factor for ROP in a population in Denmark. A similar study by Nugud et al ${ }^{39}$ also reported that 67 preterm infants in their study population had RDS, and 22 of these patients developed ROP. Respiratory complications identified in preterm infants require intervention through ventilation and oxygen supplementation to achieve postnatal stabilization. Premature infants are given oxygen supplementation while they still have an underdeveloped antioxidant system, and this causes a spurt of oxygen-free radicals, pro-oxidant disproportion, oxidative stress, and tissue damage. ${ }^{40}$ The study by Hwang et $\mathrm{a}^{32}$ also observed a correlation between longer duration of invasive ventilation and oxygen supplementation to address respiratory complications with ROP. Invasive ventilation was indicated as a confounding variable to excessive oxygen supplementation in respiratory distress and is associated with ROP. However, Colaizy et $\mathrm{al}^{41}$ established that standardized oxygen therapy plays an important role in preventing the progression of stage II ROP, thereby minimizing the risk of permanent visual loss in children born prematurely.

The identification of 38 infants with a birth weight of above $2500 \mathrm{~g}$ with ROP shows that low birth weight/gestational age is not the only factor leading to ROP development. This shows that screening should extend to infants with more than $1500 \mathrm{~g}$ birth weight or gestational age of more than 37 weeks with an unstable clinical course. The two years follow-up revealed higher incidences of subnormal vision and strabismus in preterm children than in full-term cohort. This result is similar to that of Monika et $\mathrm{al}^{42}$ who found that ROP is associated with poorer vision later in life.

From the present study, we found that the risk of ROP increased with the decrease in birth weight decreased. This observation is similar to previous studies. However, our study found the aHR of ROP for RDS children dropped to 1.07 or lower for infants with birth weights of less than $1500 \mathrm{gm}$, not significant when compared to non-RDS children at the corresponding birth weight. It is clear that the lower the birth weight, the higher the risk of developing ROP, even for non-RDS children. The incidence of ROP does not increase, in less than $1000 \mathrm{~g}$, in the RDS group compared to the control. However, for infants with the birth weights less than $1500 \mathrm{~g}$, the RDS cohort to the comparison cohort aHRs was not significant. There are probably other risk factors that influence the appearance of ROP that were not analyzed in the study.

A longer length of stay in the NICU is significantly associated with higher hazards of developing ROP. This study revealed a higher incidence of ROP for infants $(\mathrm{n}=1231$ or $16.3 \%)$ who had a stay at hospital for 30 days or longer, indicating severe ROP. NICU care is associated with hyperoxia, an excessive supply of oxygen that suppresses growth factors once the infants are placed there. Consequently, the poorly vascularized yet increasingly metabolically active retina becomes hypoxic, triggering the growth factor-induced proliferation within blood vessels, thereby increasing the risk of retinal detachment and blindness. ${ }^{8}$ This result is similar to the finding in the US study that a LOS greater than 28 days significantly increased the risk of developing ROP as compared to normal birth weight. ${ }^{15}$ A longer length of stay indicates an unstable clinical course for premature infants, and complications increase the risk for ROP.

This study revealed that boys were at a higher risk of developing ROP than girls. This finding is similar to other studies. ${ }^{31,34,35}$ Male sex has been especially linked to worse outcomes of respiratory comorbidities and these increase the risk of ROP. ${ }^{35-37}$ Our study failed to show this characteristic.

When a preterm infant with RDS is resuscitated in high oxygen concentrations, it leads to saturation in arterial oxygen and higher than normal oxygen saturation levels are linked to the damage of newly developed retinal capillaries. ${ }^{2}$ This study established a correlation between respiratory complications and ROP. ROP was established in preterm infants who received both invasive as well as non-invasive respiratory support. In our study, approximately half of ROP cases occurred in preterm infants who received invasive respiratory support at birth. The relationship between mechanical ventilation uses and ROP development has been well studied. ${ }^{38-41}$ Invasive ventilation was indicated as a confounding variable to excessive oxygen supplementation in respiratory distress, and is associated with ROP. However, Colaizy et $\mathrm{al}^{41}$ established that standardized oxygen therapy plays an important role in preventing the progression of stage II ROP, thereby minimizing the risk of permanent visual loss in children born prematurely.

Treatment for ROP is based on disease severity. ${ }^{38-41}$ Most infants with stages 1 or 2 ROP eventually improve without treatment. Treatment is initiated once the infant develops type I ROP. Areas of the retina may be treated with cryotherapy or laser therapy to prevent or reverse the proliferation of abnormal retinal vessels, thereby reducing complications (eg, retinal detachment) and preserving central vision. Pharmacological therapies with intra virtual injections of anti-vascular endothelial growth factor (VEGF) agents (eg, Avastin or Lucentis) are currently being used as an alternative to, or in 
addition to, laser treatment. However, if the ROP continues to worsen, treatment with vitrectomy and/or a scleral buckling procedure is always needed. Our data showed that only $18(1.08 \%)$ children in the RDS cohort and $6(0.52 \%)$ in comparisons required treatment of scleral buckling or vitrectomy.

\section{Study Significance and Implications}

ROP is a major cause of preventable childhood blindness. In Taiwan, the average occurrence of ROP was $36.6 \%$, indicating that a large number of premature infants are at the risk of visual impairment. The advancement in neonatal intensive care translates into an increase in the number of immature new-born who survives. This directly increases the number of preterm infants at risk of developing ROP. Therefore, understanding the risk factors associated with ROP occurrence within the local context can help in their modification, and to prevent or/and minimize the incidence of this vision-threatening disorder. While most studies are hospital-based, this study followed up the study cohorts for one year. Our study will add on to the limited number of studies and knowledge on the risk factors associated with ROP in Taiwan.

The findings of this study provide clinical implications for developing therapeutic strategies for ROP prevention to reduce disease progression. The results of this study are consistent with those of other studies that linked the incidence and severity of ROP to birth weight. This population-based study indicates respiratory distress is an important factor. Clinicians caring for preterm infants in Taiwan should be aware of the risk factors in this population, and work towards minimizing them to prevent ROP. This study recommends that the ROP screening practice should be extended to preterm infants with a normal birth weight. Besides, a proper balance should be established between neonatal intensive care provisions such as oxygen supplementation so that this necessary support for life does not produce adverse effects such as ROP. This study also recommends that children with ROP get life-long ophthalmological follow-up.

This study results also imply that further studies should be conducted to explore the actual mechanism that invasive/ mechanical ventilation increased the risk of ROP. Additionally, studies should further explore the reason for the higher ROP incidence in preterm infants born to urban or white-collar parents.

\section{Study Strengths and Limitations}

The strength of this study is using a large sample size obtained from the National Health Insurance infant population database. The large sample size made it possible to statistically evaluate several risk factors, and enhance the reliability, generalizability, and transferability of the study results. This study was able to extract the classification of ROP in the identified cases and thus allowed to evaluate severity of retinopathy of prematurity. There are several limitations. First, the databases of mothers and newborns belong to different databases in the health insurance database. Because newborns do not have ID cards, many key medical and infant characteristics are initially archived in the mother's database, making it impossible to collect and analyze newborn data in the children's database. This study uses the children's database for analysis; some infant characteristics were unavailable to adjust for these potential confounders in the data analysis. Second, this study is the retrospective nature, as the researchers were not in control of the exposures or outcome assessments, but had to rely on the recordkeeping based on insurance claims. About $25 \%$ of the preterm infants were excluded from this study, mainly because information on birth weight and length of stay at hospital was unavailable. A portion of 258 babies with birth weights $<1000 \mathrm{~g}$ in the non-RDS cohort might be children of RDS, but not diagnosed. The overall risk for RDS might be thus underestimated. Retrospective data collection brings out the issue of data validity. Nonetheless, the one-year follow-up allowed us to evaluate the relationship between RDS and the ROP risk.

\section{Conclusion}

Our findings show that in addition to respiratory distress, low birth weight, length of stay in the NICU and female gender also play important role in the development of ROP. Although low birth weight and female gender are non-modifiable factors, they aid in identifying preterm infants at an increased risk of ROP. Thus, they should be monitored closely to diagnose the disease early enough for effective treatment interventions. Prolonged stay in the NICU under ventilation and supplemental oxygen supply can be modified to equivalents that favor proper retina vascularization. Therefore, it is imperative to examine the extent of the vascularized retina in children born prematurely and to regularly screen the retina to ensure the retina vascularizes normally. The incidence of ROP in Taiwan is quite high in premature infants, and proper 
interventions through screening, adjusting modifiable risk factors and treatments are necessary to prevent childhood vision disorders and reduce the high cost of hospitalization that are associated with ROP. However, children with ROP require life-long follow-up as their vision may develop ocular morbidities later in life.

\section{Abbreviations}

RDS, Respiratory Distress Syndrome; ROP, Retinopathy of prematurity; aHR, adjusted hazard ratios; NICU, neonatal intensive care unit; ICD-9-CM, International Classification of Diseases, 9th Revision Clinical Modification; 95\% CI, 95\% confidence interval; LBW, low birth weight.

\section{Data Sharing Statement}

We accessed the database from the Taiwan National Health Research Institutes. We are not eligible to duplicate and disseminate the database. For further access to the database, please contact the Ministry of Health and Welfare (Email: stcarolwu@mohw.gov.tw) for assistance. Taiwan Ministry of Health and Welfare Address: No.488, Sec. 6, Zhongxiao E. Rd., Nangang Dist., Taipei City 115, Taiwan. Phone: +886-2-8590-6848.

\section{Ethics Approval and Consent to Participate}

All identification numbers were replaced with surrogate numbers for insured people to protect the privacy. This study was approved by the Research Ethics Committee of China Medical University and Hospital (CMUH104-REC2-115). No consents are required from the study population.

\section{Author Contributions}

All authors have made a significant contribution to this study, all the way through the conception, study design, execution, acquisition of data, data analysis and interpretation to drafting, revising or critically reviewing stages of the article. The authors also gave final approval of the version to be published, agreed on the journal to which the article has been submitted, and agreed to be accountable for all aspects of the work.

\section{Funding}

This study was partly supported by the Ministry of Health and Welfare, Taiwan (MOHW109-TDU-B-212-114004), MOST Clinical Trial Consortium for Stroke (MOST 109-2321-B-039-002), the Ministry of Science and Technology, Taiwan (Grant no. MOST108-2410-H-039-001 and MOST109-2410-H-039-001), the China Medical University, Taiwan (Grant no. CMU109-MF-119), and Tseng-Lien Lin Foundation, Taichung, Taiwan.

\section{Disclosure}

The authors declare no competing interests in this work.

\section{References}

1. Choi JY, Han YI, Kim JH, Kim ES, Jeon J. The most important factors for retinopathy of prematurity in preterm infants. Korean J Perinatol. 2014;25:3. doi:10.14734/kjp.2014.25.3.153

2. Hartnett ME, Lane RH. Effects of oxygen on the development and severity of retinopathy of prematurity. J AAPOS. 2013;17:229-234. doi:10.1016/j. jaapos.2012.12.155

3. Hellstrom A, Smith LEH, Dammann O. Retinopathy of prematurity. Lancet. 2013;382:1445-1457. doi:10.1016/S0140-6736(13)60178-6

4. Feghhi M, Altayeb SM, Haghi F, et al. Incidence of retinopathy of prematurity and risk factors in the South-Western region of Iran. Middle East Afr J Ophthalmol. 2012;19:101-106. doi:10.4103/0974-9233.92124

5. Hadi AM, Hamdy IS. Correlation between risk factors during the neonatal period and appearance of retinopathy of prematurity in preterm infants in neonatal intensive care units in Alexandria, Egypt. Clin Ophthalmol. 2013;7:831-837. doi:10.2147/OPTH.S40136

6. Reyes ZS, Al-Mulaabed SW, Bataclan F, et al. Retinopathy of prematurity: revisiting incidence and risk factors from Oman compared to other countries. Oman J Ophthalmol. 2017;10:26-32. doi:10.4103/ojo.OJO_234_2014

7. VanderVeen DK, Bremer DL, Fellows RR, et al. Prevalence and course of strabismus through age six years in participants of the early treatment for retinopathy of prematurity randomized trial. J AAPOS. 2011;15:536-540. doi:10.1016/j.jaapos.2011.07.017

8. Freitas AM, Mörschbächer R, Thorell MR, Rhoden EL. Incidence and risk factors for retinopathy of prematurity: a retrospective cohort study. Int $J$ Retin Vitr. 2018;4:20. doi:10.1186/s40942-018-0125-z 
9. Celebi AR, Petricli IS, Hekimoglu E, Demirel N, Bas AY. The incidence and risk factors of severe retinopathy of prematurity in extremely low birth weight infants in Turkey. Med Sci Monit. 2014;20:1647-1653. doi:10.12659/MSM.892262

10. Blencowe H, Lawn JE, Vazquez T, Fielder A, Gilbert C. Preterm-associated visual impairment and estimates of retinopathy of prematurity at regional and global levels for 2010. Pediatr Res. 2013;74:35-49. doi:10.1038/pr.2013.205

11. Fierson WM, Saunders RA, Good W; American Association for Pediatric Ophthalmology and Strabismus, American Association of Certified Orthoptists. Screening examination of premature infants for retinopathy of prematurity. Pediatrics. 2013;131:189-195. doi:10.1542/peds.2012-2996

12. Holmström G, Larsson E. Outcome of retinopathy of prematurity. Clin Perinatol. 2013;40:311-321. doi:10.1016/j.clp.2013.02.008

13. Painter SL, Wilkinson AR, Desai P, Goldacre MJ, Patel CK. Incidence and treatment of retinopathy of prematurity in England between 1990 and 2011: database study. Br J Ophthalmol. 2015;99:807-811. doi:10.1136/bjophthalmol-2014-305561

14. VanSorge A, Termote J, Kerkhoff $\mathrm{F}$, et al. Nationwide inventory of risk factors for retinopathy of prematurity in the Netherlands. $J$ Pediatr. 2014;164:494-498.e1. doi:10.1016/j.jpeds.2013.11.015

15. Ludwig CA, Chen TA, Hernandez-Boussard T, Moshfeghi AA, Moshfeghi DM. The epidemiology of retinopathy of prematurity in the United States. Ophthalmic Surg Lasers Imaging Retina. 2017;48:553-562. doi:10.3928/23258160-20170630-06

16. Sahin A, Sahin M, Türkcü FM, et al. Incidence of retinopathy of prematurity in extremely premature infants. ISRN Pediatr. 2014;2014. doi:10.1155/2014/134347

17. Kang EYC, Lien R, Wang NK, et al. Retinopathy of prematurity trends in Taiwan: a 10-year nationwide population study. Invest Ophthalmol Vis Sci. 2018;59:3599-3607. doi:10.1167/iovs.18-2402

18. Eckert G, Fortes Filho JB, Maia M, Procianoy RS. A predictive score for retinopathy of prematurity in very low birth weight preterm infants. Eye. 2012;26:400-406. doi:10.1038/eye.2011.334

19. Binenbaum G. Algorithms for the prediction of retinopathy of prematurity based on postnatal weight gain. Clin Perinatol. 2013;40:261-270. doi:10.1016/j.clp.2013.02.004

20. Gonçalves E, Násser LS, Martelli DR, et al. Incidence and risk factors for retinopathy of prematurity in a Brazilian reference service. Sao Paulo Med J. 2014;132:85-91. doi:10.1590/1516-3180.2014.1322544

21. Akkawi MT, Shehadeh MM, Shams ANA, et al. Incidence and risk factors of retinopathy of prematurity in three neonatal intensive care units in Palestine. BMC Ophthalmol. 2019;19:189. doi:10.1186/s12886-019-1180-4

22. Holmstrom G, Broberger U, Thomassen P. Neonatal risk factors for retinopathy of prematurity - a population based study. Acta Ophthalmol Scand. 1998;76:204-207. doi:10.1034/j.1600-0420.1998.760216.x

23. Akkoyun I, Oto S, Yilmaz G, et al. Risk factors in the development of mild and severe retinopathy of prematurity. J AAPOS. 2006;10:449-453. doi:10.1016/j.jaapos.2006.05.007

24. Lin HJ, Lin CC, Tsai SW, Lin HC, Su BH. Risk factors for retinopathy of prematurity in very low birth-weight infants. $J$ Chin Med Assoc. 2003;66:662-668.

25. Moral-Pumarega MT, Caserío-Carbonero S, De-la-cruz-bértolo J, Tejada-Palacios P, Lora-Pablos P, Pallás-Alonso CR. Pain and stress assessment after retinopathy of prematurity screening examination: indirect ophthalmoscopy versus digital retinal imaging. BMC Pediatr. 2012;12:132. doi:10.1186/1471-2431-12-132

26. Dyer J. Neonatal respiratory distress syndrome: tackling a worldwide problem. Pharm Ther. 2019;44:2-14.

27. Harker J, Jaramillo ML. Colombian neonatal research network. Very early surfactant without mandatory ventilation in premature infants treated with early continuous positive airway pressure: a randomized, controlled trial. Pediatrics. 2009;123:137-142. doi:10.1542/peds.2007-3501

28. Shi Y, Muniraman H, Biniwale M, Ramanathan R.A review on non-invasive respiratory support for management of respiratory distress in extremely preterm infants. Front Pediatr. 2020;8:270. doi:10.3389/fped.2020.00270

29. Beharry KD, Valencia GB, Lazzaro DR, Aranda JV. Pharmacologic interventions for the prevention and treatment of retinopathy of prematurity. Semin Perinatol. 2016;40:189-202. doi:10.1053/j.semperi.2015.12.006

30. Sankar MJ, Sankar J, Chandra P. Anti-vascular endothelial growth factor (VEGF) drugs for treatment of retinopathy of prematurity. Cochrane Database Syst Rev. 2018;1:CD009734. doi:10.1002/14651858.CD009734.pub2

31. Lundgren P, Kistner A, Andersson EM, et al. Low birth weight is a risk factor for severe retinopathy of prematurity, depending on gestational age. PLoS One. 2014;9:e109460. doi:10.1371/journal.pone.0109460

32. Hwang JH, Lee EH, Kim EA. Retinopathy of prematurity among very-low-birth-weight infants in Korea: incidence, treatment, and risk factors. $J$ Korean Med Sci. 2015;30:S88-S94. doi:10.3346/jkms.2015.30.S1.S88

33. Glass HC, Costarino AT, Stayer SA, Breet CM, Cladis F, Davis PJ. Outcome for extremely premature infants. Anesth Analg. 2015;120:1337-1351. doi:10.1213/ANE.00000000000070

34. Lorente-Pozo S, Parra-Llorca A, Torres B, et al. Influence of sex on gestational complications, fetal-to-neonatal transition, and postnatal adaptation. Front Pediatr. 2018;6:63. doi:10.3389/fped.2018.00063

35. Shim SY, Cho SJ, Kong KA, Park EA. Gestational age-specific sex difference in mortality and morbidities of preterm infants: a nationwide study. Sci Rep. 2017;7:6161. doi:10.1038/s41598-017-06490-8

36. Binet ME, Bujold E, Lefebvre F, Tremblay T. Role of gender in morbidity and mortality of extremely premature neonates. Am J Perinatol. 2012;29:159-166. doi:10.1055/s-0031-1284225

37. Lingappan K, Jiang W, Wang L, Moorthy B. Sex-specific differences in neonatal hyperoxic lung injury. Am J Physiol Lung Cell Mol Physiol. 2016;311:L481-L493. doi:10.1152/ajplung.00047.2016

38. Slidsborg C, Jensen A, Forman JL, et al. Neonatal risk factors for treatment-demanding retinopathy of prematurity. Ophthalmol. 2016;123:796-803. doi:10.1016/j.ophtha.2015.12.019

39. Nugud AA, Nugud S, Nugud A, Assmaa AN, Kathamuthu R, Jalal M. Perinatal risk factors for development of retinopathy of prematurity in a tertiary neonatal intensive care unit. J Taibah Univ Medical Sci. 2019;14:306-311. doi:10.1016/j.jtumed.2019.05.001

40. Vento M. Oxygen supplementation in the neonatal period: changing the paradigm. Neonatology. 2014;105:323-331. doi:10.1159/000360646

41. Colaizy TT, Longmuir S, Gertsch K, Abràmoff MD, Klein JM. Use of a supplemental oxygen protocol to suppress progression of retinopathy of prematurity. Investig Ophthalmol Vis Sci. 2017;58(2):887-891. doi:10.1167/iovs.16-20822

42. Monika E, Anna S, Cecilia K, et al. A Comparison of Macular Structure Imaged by Optical Coherence Tomography in Preterm and Full-Term Children. Investigative Ophthalmology \& Visual Science. November 2007, Vol.48, 5207-5211. doi:10.1167/iovs.06-1199 


\section{Publish your work in this journal}

The International Journal of General Medicine is an international, peer-reviewed open-access journal that focuses on general and internal medicine, pathogenesis, epidemiology, diagnosis, monitoring and treatment protocols. The journal is characterized by the rapid reporting of reviews, original research and clinical studies across all disease areas. The manuscript management system is completely online and includes a very quick and fair peer-review system, which is all easy to use. Visit http://www.dovepress.com/testimonials.php to read real quotes from published authors.

Submit your manuscript here: https://www.dovepress.com/international-journal-of-general-medicine-journal 